\title{
Outcome of Irrigation and Debridement after Failed Two-Stage Reimplantation for Periprosthetic Joint Infection
}

\author{
M. Faschingbauer $\mathbb{D}^{1,}{ }^{1,2}$ F. Boettner, ${ }^{2}$ R. Bieger, ${ }^{1}$ C. Weiner, ${ }^{1}$ H. Reichel, ${ }^{1}$ and T. Kappe \\ ${ }^{1}$ Department for Orthopaedic Surgery, RKU, University of Ulm, Oberer Eselsberg 45, 89081 Ulm, Germany \\ ${ }^{2}$ Hospital for Special Surgery, 535 East 70th Street, New York, NY 10021, USA \\ Correspondence should be addressed to M. Faschingbauer; martin.faschingbauer@outlook.com
}

Received 29 March 2018; Revised 14 July 2018; Accepted 5 September 2018; Published 11 October 2018

Guest Editor: Bernd Fink

Copyright (c) 2018 M. Faschingbauer et al. This is an open access article distributed under the Creative Commons Attribution License, which permits unrestricted use, distribution, and reproduction in any medium, provided the original work is properly cited.

\begin{abstract}
Introduction. Two-stage revision is the gold standard for the treatment of deep implant infection after knee or hip arthroplasty. Irrigation and debridement may be a treatment option for failed 2-stage revisions in cases where a reinfection occurs within 30 days or the symptoms exist not longer than 3 weeks and is appealing because of its low morbidity. We determined the incidence of recurrent infections following irrigation and debridement for failed two-stage revision hip and knee arthroplasty. Methods. We performed a single center retrospective review of periprosthetic hip and knee infections treated with a two-stage procedure from 2002 to 2010. All patients that subsequently underwent irrigation and debridement for a subsequent infection were selected for the current study. Results. 440 two-stage revisions were performed between 2002 and 2010. Fifty-one two-stage revisions failed (11.6\%). Nineteen failed two-stage revisions were treated with irrigation and debridement; 12 (63.2\%) patients remained free of infection at follow-up (mean follow-up: 39 months; range, 24-90 months), infection persisted in 6 patients (31.6\%), and 1 patient died (5.3\%). Conclusions. Success rates of irrigation and debridement for failed two-stage procedures are similar to the success rates of irrigation and debridement in primary implant infections. According to the current paper, irrigation and debridement are an acceptable treatment for acute reinfections after failed two-stage revision if performed within the first 30 postoperative days after failed two-stage procedure or if symptoms are present for less than 3 weeks in the presence of a susceptible organism.
\end{abstract}

\section{Introduction}

Periprosthetic infection after total joint replacement is a devastating complication and occurs in up to $2 \%$ of primary joint replacements [1-5]. Treatment options include irrigation and debridement (I\&D) with component retention [610], one-stage revision [11-13], or two-stage revision utilizing an antibiotic containing cement spacer [14-16].

Two-stage revision remains the golden standard treatment in the United States with infection-free survival rates of $80-100 \%[17-19]$. An antibiotic containing cement spacer [14, 16] and intravenous antibiotics are routinely used for twostage procedures. Zimmerli et al. [20] recommended twostage revisions for patients with symptoms for more than 3 weeks or an index procedure performed more than 30 days ago.

Patients presenting with symptoms for less than 3 weeks or within 30 days of primary joint replacement without a sinus tract or radiographic evidence of component loosening can be considered for an I\&D with retention of components in case of and a susceptible organism [21]. This approach is attractive because of its low-morbidity and cost effectiveness. However, failure rate of 7-79\% has been reported and I\&D is therefore in general considered less effective than two stage revision $[6,22]$.

There are much less clear recommendations for the treatment of recurrent infection following two stage revisions. Whiteside et al. [23] reported control of infection in 17 of 18 patients using aggressive I\&D and intraarticular antibiotic infusion over 6 weeks after a failed two-stage treatment attempt.

The aim of the current study was to evaluate the clinical success rates of I\&D after failed two-stage revision and to investigate the impact of the type of organism, patient age, general health condition (ASA), and comorbidities on treatment outcome. 


\section{Materials and Methods}

The current study is a retrospective chart review of 440 two-stage revisions for periprosthetic knee or hip infection performed at one tertiary referral center between 2002 and 2010.

Patient demographics including age, gender, BMI, health status (ASA), the timespan between $I \& D$, and the prior failed two-stage procedure, as well as number and type of prior surgeries, were recorded. The American Society of Anesthesiologists (ASA) physical status classification score was used as a proxy variable for health status. The types of organisms and sensitivities were documented for all procedures. Quinolone resistant Gram-negative bacteria, rifampicin-resistant Staphylococcus, Enterococcus, and Candida were classified as "difficult to treat" (DTT) in accordance of Winkler et al. [27].

The diagnosis of infection prior to the index two-stage procedure was based on clinical signs, blood work (ESR, CRP), positive synovial fluid aspiration, and intraoperative cultures (following the state of art [28]). The index two-stage revisions included removal of implants and bone cement (Stage I). All patients received a static antibiotic containing cement spacer (knees) or an articulating spacer (hip) as well as systemic antibiotics based on organism sensitivity for 2 weeks intravenously and for an additional 4 weeks orally. The articulating hip spacers were performed as mould-spacers with an endoskeleton (67\%) or as handmade spacers (33\%) [29]. The knee spacers were performed as static, handmade spacers with an endoskeleton [30]. Two weeks after stopping the systemic antibiotics successful eradication was confirmed by repeat joint aspiration. If the aspiration was negative, CRP remained less than $2 \mathrm{~g} / \mathrm{dL}$ and there was no sinus tract and a new implant was inserted (Stage II).

Reinfection occurred in 51 patients (11.6\%) of 440 twostage revisions. If a reinfection occurred (diagnosis of reinfection followed the consensus criteria by Zmistowski et al. [1]) within 30 days after two-stage revision or patients presented with an acute reinfection (symptoms for less than 3 weeks), aggressive, if necessary repeated I\&D was performed. Nineteen of 51 patients fulfilled the inclusion criteria (32 patients were treated with a second two-stage procedure). The mean age at I\&D after failed two-stage procedure was 67.3 years (range, 45.2 - 84.5 years); there were 12 male and 7 female patients, 12 hips and 7 knees, 10 left and 9 right joints; the mean BMI was $29.6 \mathrm{~kg} / \mathrm{m}^{2}$ (range, 21.5 to $36.4 \mathrm{~kg} / \mathrm{m}^{2}$ ). Mean follow-up was 39 months (range, 24-90 months). At the time of each follow-up the absence of infection was evaluated by clinical criteria (no sinus tract, no swelling, no erythema, and no tenderness); if there was a reasonable suspicion of recurrent infection, an immediate work-up was done adhering to the guidelines by Parvizi et al. [28].

I\&D was performed utilizing the preexisting incisions. After a thorough synovectomy and irrigation of the surgical site all removable components (hip: head and liner; knee: articular insert) were removed. Now the implant was cleaned and a through irrigation utilizing a minimum of $10 \mathrm{~L}$ of antiinfectious irrigation was performed. Finally the surgical field was covered with new drapes, new instruments were opened, and the surgeons and assistance were regloved and regowned. New mobile components were put in place and the wound was closed in layers in a usual way. If the soft tissues were not stable enough, 1-3 polyurethane sponges were inserted under the fascia or subcutaneously and were connected to a vacuum producing devise via tubes [31]; afterwards the wounds were still closed in layers under meticulous reconstruction and accurate adaptation of the tissue layers. A repeated I\&D was performed 3-6 days later in a technique described by Kelm et al. [31]. The indication for a repetition of I\&D was evidence of persistent microorganism intraoperatively, persisting drainage, no decrease of $\mathrm{C}$-reactive protein within 6 days combined with clinical signs of persistent infection (overheat, reddening), or persisting sepsis.

All patients terminated the use of antibiotics two weeks after last surgery ("postoperative antibiotics", Tables 2 and 4), and no suppression therapy was used.

Failure was defined as any additional surgery due to infection after hospital discharge. Only patients with a minimum follow-up of 24 months were included in the current study.

Descriptive statistics were calculated, including mean and frequency. Mann-Whitney test was used to determine differences between successes and failures. Chi-square tests were used to determine differences in proportions between dichotomous data.

All statistical analyses were performed using IBM SPSS Statistics software version 23 (Armonk, NY: IBM Corp.). A p-value of less than 0.05 was considered to be statistically significant.

\section{Results}

Of 19 patients who underwent I\&D for failed two-stage revision, $12(63.2 \%)$ patients were infection-free after a minimum follow-up of 24 months (mean 39 months, range 24-90 months). A recurrent deep infection occurred in 6 patients (31.6\%) and one patient died (5.3\%). 8/11 hips (72.7\%) and 4/7 (57.1\%) knees were infection-free after the minimum followup.

Patients with successful (Tables 1 and 2) and failed treatment (Tables 3 and 4) differed in regard to BMI (median, success group $31.5 \mathrm{~kg} / \mathrm{m}^{2}$, reinfection group $25.5 \mathrm{~kg} / \mathrm{m}^{2}, \mathrm{p}=$ $0.026)$, but there were no differences in age $(\mathrm{p}=0.892)$, ASA grade $(\mathrm{p}=0.989)$, and number of I\&Ds $(\mathrm{p}=0.243)$.

Causative organisms cultured at the time of I\&D are reported in Tables 2 and 4. "Difficult to treat" organisms occurred in 6 cases. Two polymicrobial infections were observed. No statistically significant difference was found concerning the distribution of DTT-organisms or polymicrobial infections between the successfully treated and failed group.

\section{Discussion}

Periprosthetic infections are a devastating complication. Treatment options range from two-stage revision [22], onestage exchange [11], or irrigation and debridement with retention of components [8]. There is an ongoing debate about the most appropriate treatment of an acute implant infection. 
TABLE 1: Successful cases and demographics.

\begin{tabular}{|c|c|c|c|c|c|c|c|c|}
\hline Patient & Age (years) & Gender & Joint & Side & ASA grade & Follow up (months) & BMI & Comorbidities \\
\hline 1 & 73.5 & male & Hip & $\mathrm{R}$ & 3 & 33 & 34.2 & HTN, A \\
\hline 2 & 46.5 & male & Hip & $\mathrm{R}$ & 3 & 34 & 32.1 & HTN, SM, A \\
\hline 3 & 68.3 & male & Hip & $\mathrm{L}$ & 3 & 28 & 30.9 & HTN, CKF \\
\hline 7 & 74.4 & female & Hip & $\mathrm{L}$ & 3 & 63 & 36.4 & HTN, post enterovesical fistula \\
\hline 8 & 63.2 & male & Hip & $\mathrm{L}$ & 2 & 77 & 21.5 & SM, DM \\
\hline 9 & 64.3 & female & Hip & $\mathrm{R}$ & 2 & 24 & 35.4 & $\mathrm{HTN}, \mathrm{CHF}$ \\
\hline 11 & 52.9 & female & Hip & $\mathrm{L}$ & 2 & 90 & 34.3 & - \\
\hline 12 & 75.9 & female & Knee & $\mathrm{R}$ & 3 & 24 & 32.8 & $\begin{array}{c}\text { HTN, absolute arrhythmia in } \\
\text { atrial fibrillation }\end{array}$ \\
\hline 13 & 45.2 & male & Knee & $\mathrm{R}$ & 2 & 25 & 29.3 & Post ORIF femur \\
\hline 14 & 76.2 & male & Knee & $\mathrm{R}$ & 3 & 30 & 29.8 & $\begin{array}{l}\text { HTN, CHD, CHF, CKF, absolute } \\
\text { arrhythmia in atrial fibrillation }\end{array}$ \\
\hline 18 & 84.5 & male & Hip & $\mathrm{L}$ & 4 & 28 & 24.2 & $\begin{array}{l}\text { HTN, CHD, CKD, post apoplexy, } \\
\text { morbus parkinson, chronic } \\
\text { cystitis }\end{array}$ \\
\hline 19 & 67.4 & female & Knee & $\mathrm{R}$ & 3 & 27 & 29.7 & $\begin{array}{l}\text { Bipolar psychosis, lumbar spinal } \\
\text { syndrome }\end{array}$ \\
\hline
\end{tabular}

$\mathrm{DM}=$ diabetes mellitus, $\mathrm{HTN}=$ arterial hypertension, $\mathrm{CHD}=$ coronary heart disease, $\mathrm{CHF}=$ chronic heart failure, $\mathrm{CKF}=$ chronic kidney failure, $\mathrm{COPD}=$ chronic obstructive pulmonary disease, $\mathrm{SM}=$ smoker, $\mathrm{A}=$ alcohol $(>20 \mathrm{~g} / \mathrm{d}), \mathrm{DA}=$ drug abuse

While eradication rates are higher in two-stage procedures, quality of life and postoperative function might be better after one-stage and I\&D procedures, respectively [32-34].

Eradication rates following revision of primary implant infections range from 61 to $100 \%$ for different treatment protocols $[25,32,33]$. However, literature on the treatment of recurrent infection is scarce. The current study reports the eradication rate utilizing I\&D for recurrent periprosthetic infection within a time-window of 30 days or occurring symptoms less than 3 weeks in patients with failed two-stage revision for periprosthetic infection. In the present study similar eradication rates for an I\&D (63.2\%) after failed twostage revision were shown compared to I\&D in primary deep implant infection $[6,35,36]$.

The current study has the following limitations. First, this is a retrospective study. Second, while the report is based on a large group of patients undergoing two-stage revision surgery cases, numbers in the current group of I\&D for recurrent infection are too small to analyze the impact of the type of organism and its sensitivity on overall outcome of I\&D. The numbers are also too small to make a differentiated analysis between hips and knees. Third, since patients did not undergo laboratory screening or recurrent aspiration, infections were assumed to be eradicated based on clinical criteria exclusively. Finally, the use of a polyurethane sponge with a vacuum-producing device during repeated I\&Ds is not very well described in the literature. There are some reports using V.A.C-Instill with small patient-numbers, but only one study, in which also a deep sponge was placed and the wound was still closed anatomically [31] as in the current study. In our center the use of sponges with a vacuum system was left after 2010.
There are only a few papers that report on the treatment of failed treatment attempts of deep implant infections. There is no common sense of the best treatment after failed two-stage procedure. Stammers et al. [24] reported failure rates of $42 \%$ (8/19 knees) (Table 5) for repeat two stage revision after failed initial two stage treatment.

There is a study using a decision tree analysis to determine the best treatment (quality of life) after failed revision for deep implant infection. Wu et al. [37] expected the highest QoL utilizing arthrodesis following a failed two-stage revision in patients with total knee replacement. In a clinical review Sherrell et al. [25] reported failure rates of $34 \%$ in twostage revisions for patients who underwent I\&D followed by two-stage revision due to persisting infection. The authors assumed that the failure rate of $34 \%$ is higher than in patients who undergo two-stage revision only (Table 5).

Pagnano et al. [26] reported a reinfection rate of $18.7 \%$ (27/144 hips) after a first two-stage revision due to periprosthetic infection. The authors reported four treatment options after failed first two-stage revision: antibiotic suppression therapy, I\&D, resection arthroplasty, or a second two-stage revision. Two of 3 patients did not need any further surgery after I\&D and continuous oral suppressive antibiotic therapy. Sixteen patients were treated by resection arthroplasty after failed two-stage procedure. Three of these 16 patients (18.8\%) had to undergo further surgeries for recurrence of infection. Eleven patients underwent a second two-stage revision, in 8 patients $(72.7 \%)$, a recurrence of infection occurred and further surgeries were needed (Table 5).

In the current study, if necessary, repeat I\&D was performed (range, 1-10). Kelm et al. [31] reported an eradication rate of $92.9 \%$ using the above mentioned protocol. However, 


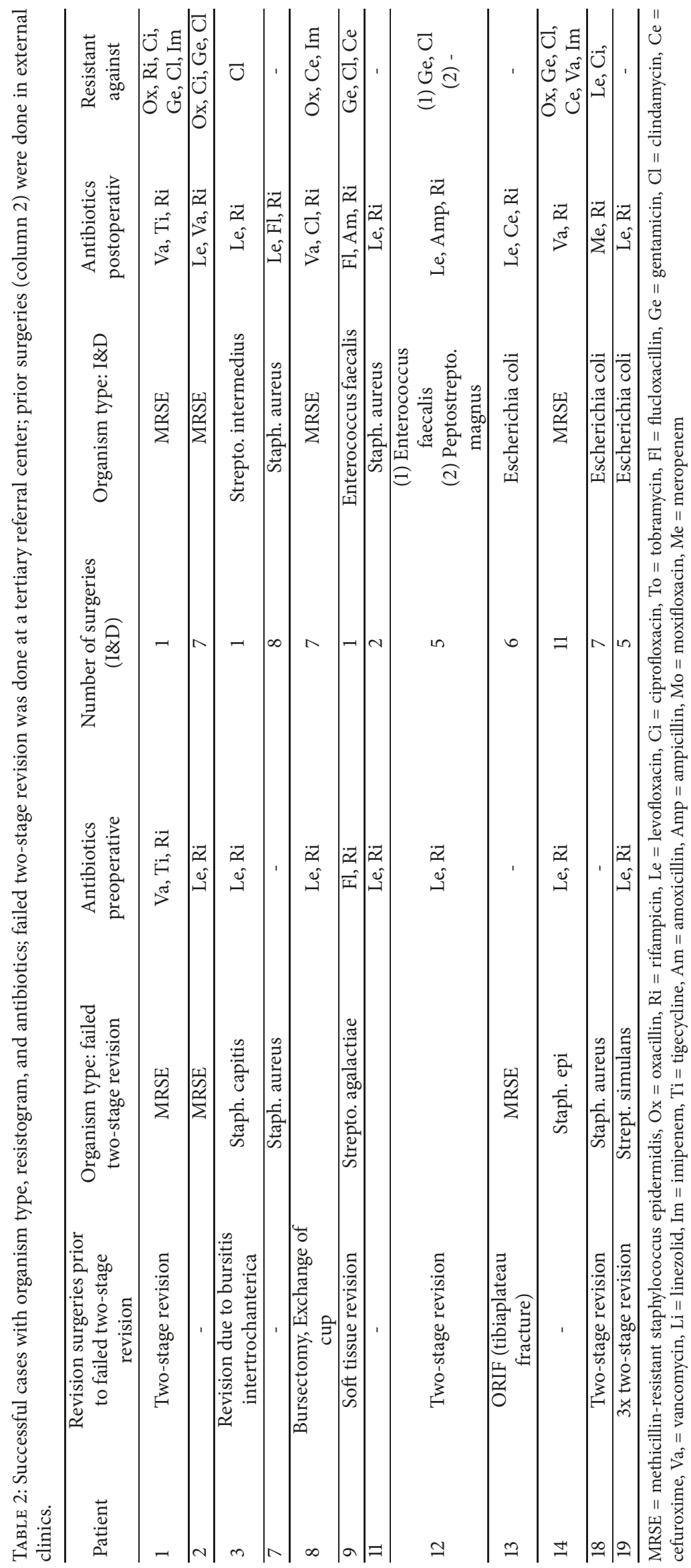


TAble 3: Failed cases and demographics.

\begin{tabular}{|c|c|c|c|c|c|c|c|c|}
\hline Patient & Age (years) & Gender & Joint & Side & ASA grade & Follow up (months) & BMI & Comorbidities \\
\hline 5 & 76.6 & female & Hip & $\mathrm{R}$ & 3 & 55 & 23.5 & $\begin{array}{c}\text { Post cerebellar infarction, } \\
\text { osteoporosis, post } \\
\text { humerus-fracture }\end{array}$ \\
\hline 6 & 70.8 & male & Hip & $\mathrm{L}$ & 3 & 29 & 27.8 & $\begin{array}{c}\text { DM, HTN, CHD, CHF, CKF, } \\
\text { post quadruple coronary artery } \\
\text { bypass, both sided carotid artery } \\
\text { stenosis }\end{array}$ \\
\hline 10 & 62.4 & male & Hip & $\mathrm{R}$ & 2 & 29 & 26.3 & $\begin{array}{c}\text { SM, A, post transient ischemic } \\
\text { attack }\end{array}$ \\
\hline 15 & 61.9 & male & Knee & $\mathrm{L}$ & 2 & 49 & 29.8 & HTN, post phlebothrombosis \\
\hline 16 & 78.5 & female & Knee & $\mathrm{L}$ & 3 & 35 & 23.5 & - \\
\hline 17 & 62.1 & male & Knee & $\mathrm{L}$ & 4 & 24 & 24.7 & $\begin{array}{l}\text { DM, HTN, CHD (bio heart } \\
\text { valve, pacemaker), CKD (IgA } \\
\text { nephropathy), A, post } \\
\text { phlebothrombosis }\end{array}$ \\
\hline
\end{tabular}

$\mathrm{DM}=$ diabetes mellitus, $\mathrm{HTN}=$ arterial hypertension, $\mathrm{CHD}=$ coronary heart disease, $\mathrm{CHF}=$ chronic heart failure, $\mathrm{CKF}=$ chronic kidney failure, $\mathrm{COPD}=$ chronic obstructive pulmonary disease, $\mathrm{SM}=$ smoker, $\mathrm{A}=\operatorname{alcohol}(>20 \mathrm{~g} / \mathrm{d}), \mathrm{DA}=$ drug abuse

the literature is inconsistent and some studies [38-40] report a higher failure rate with repeated I\&Ds.

In the current study Staphylococcus was the predominant bacterium (52.6\%; coagulase-negative staphylococci $42.1 \%$, Staphylococcus aureus 10.5\%). This finding is in line with current literature [41, 42]. "Difficult to treat"-organisms (DTT) occurred in 6 patients. Winkler et al. [27] described rifampicin-resistant Staphylococci (Patient 1), quinoloneresistant gram-negative bacteria (Patient 18), Enterococci (Patient 5, 9, 12 and perished patient), and Candida as DTTorganisms. No differences concerning the success of I\&D were observed in the present study according to the presence or absence of DTT-organisms. One polymicrobial infection (Patient 5 and 12) occurred in each group. It is not clarified yet if the classification of DTT-infections should include polymicrobial infections. Only 3 patients showed the same organism at the time of two-stage revision and I\&D (2x MRSE: Patient 1 and 2; 1x Staph. aureus: Patient 7). Two patients (Patient 14 and 15) developed methicillin-resistance (Staph. Epidermidis). Zmistowski et al. [43] differentiate between recurrence of infection (same organism at the failed two-stage procedure and at the renewed flare-up infection) and "new" reinfection with a change of microorganism. They showed a persistent infection with the same organism in $31.5 \%$. Haddad et al. [42] also reported a change of organism in reinfection in 3 of 4 (75\%) patients and specified these patients as reinfected compared to the fourth patient with a "persisting infection". Kraay et al. [44] actually described a 100\% "new" infectionrate after failed two-stage procedure (28 patients). Triantafyllopoulos et al. [45] also showed more "new" infections than persistent infections with the same microorganism. In the current study $84.2 \%$ of patients showed "new" infection. Due to the majority of "new" infections it can be concluded, as Zmistowski et al. [43] and Triantafyllopoulos et al. [45] do, that the host status with all possible comorbidities may be a major factor. The control and improvement of comorbidities cannot be overstated as the mentioned studies describe a high vulnerability of the host for a "new" infection with a high Charlson Comorbidity Index. For patients with a high Charlson Comorbidity Index as a vulnerable host and an increased risk of perioperative complications the concept of I\&D (defined as an acute reinfection within above mentioned time window) is a feasible option of treatment. On the other hand Zmistowski et al. [43] showed the only independent predictor of persistent periprosthetic joint infection was a primary infection with Staphylococcus in general, and MRSA in particular. These numbers can be shown in the current study as well as all three persistent infections occurred in patients with a Staphylococcus-infection. At the current study all persistent infections $(\mathrm{n}=3$; same microorganism within the failed two-stage procedure and the reinfection) are in the "infection free" group after I\&D. It is unclear why the success rate in persistent infections was $100 \%$. Three reasons of this circumstance could be as follows: first, the basis of the mature biofilm (prosthesis) was removed during the two-stage procedure and it might be that the revival of a mature biofilm at the new prosthesis was sufficiently stopped by a timely I\&D. Second, the numbers of patients are too small. Third, the follow-up period is too short. The antibiotic therapy used in the current study follows the guidelines by Osmon et al. [20].

In summary, the failure rate of $I \& D$ for acute recurrence of infection (within 30 days of symptoms or 3 weeks of two stage procedure) following failed two-stage treatment was $31.6 \%$ in the present study. Therefore the concept of I\&D after failed two-stage procedure might be an option in acute reinfections more than in persistent infections. The success rate is comparable to I\&D for infection of a primary joint replacement. Careful indication (see criteria), meticulous surgical debridement, and close cooperation between the microbiologist, infectious disease doctor, and surgeon are recommended. 


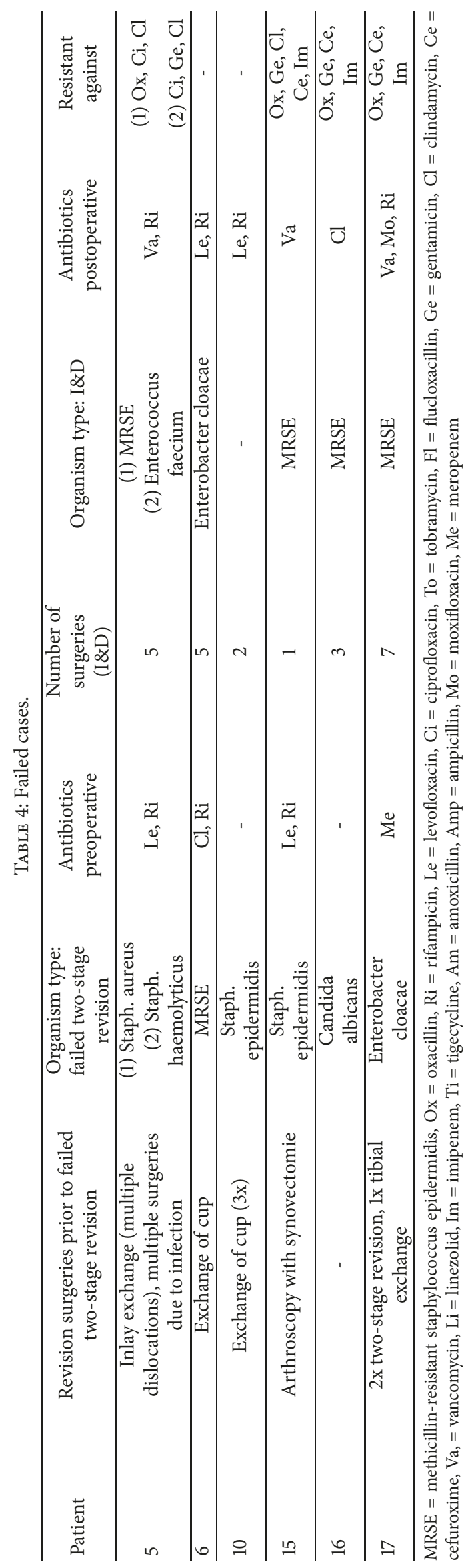


TABLE 5: Failure rates after failed revisions due to periprosthetic infection.

\begin{tabular}{lccc}
\hline Author & First Revision & Second Revision & Failure-rate \\
\hline Stammers et al. [24] & Two-stage revision & Two-stage revision & $42 \%(8 / 19)$ \\
\hline Sherrell et al. [25] & I\&D & Two-stage revision & $34 \%(28 / 83)$ \\
\hline \multirow{2}{*}{ Pagnano et al. [26] } & Two-stage revision & Resection arthroplasty & 19\% $(3 / 16)$ \\
& Two-stage revision & Two-stage revision & $73 \%(8 / 11)$ \\
\hline Current study & Two-stage revision & I\&D & $32 \%(6 / 19)$ \\
\hline
\end{tabular}

\section{Data Availability}

The data used to support the findings of this study are available from the corresponding author upon request. The paper was accepted by SICOT as a poster presentation at the 37th Orthopaedic World Congress in Rome, 2016

\section{Disclosure}

Level of Evidence is therapeutic study, Level III.

\section{Conflicts of Interest}

Dr. Boettner reports personal fees from Smith \& Nephew and Ortho Development Corporation, outside the submitted work. Dr. Faschingbauer reports personal fees from Deutsche Forschungsgemeinschaft (Research Fellowship, FA 1271/11, www.dfg.de [http://www.dfg.de]), during the conduct of the study. Neither the above-mentioned companies nor any outside organization has participated in study design or has any conflicts of interest.

\section{References}

[1] B. Zmistowski, C. D. Valle, T. W. Bauer et al., "Diagnosis of periprosthetic joint infection," Journal of Orthopaedic Research, vol. 32, no. 1, pp. S98-S107, 2014.

[2] J. Parvizi, O. F. Erkocak, and C. J. Della Valle, "Culture-negative periprosthetic joint infection," The Journal of Bone \& Joint Surgery, vol. 96, no. 5, pp. 430-436, 2014.

[3] I. S. Vanhegan, R. Morgan-Jones, D. S. Barrett, and F. S. Haddad, "Developing a strategy to treat established infection in total knee replacement," The Journal of Bone \& Joint Surgery (British Volume), vol. 94, no. 7, pp. 875-881, 2012.

[4] K. J. Bozic, E. Lau, K. Ong et al., "Risk Factors for Early Revision After Primary Total Hip Arthroplasty in Medicare Patients," Clinical Orthopaedics and Related Research, vol. 472, no. 2, pp. 449-454, 2014.

[5] S. M. Kurtz, E. Lau, J. Schmier, K. L. Ong, K. Zhao, and J. Parvizi, "Infection burden for hip and knee arthroplasty in the United States," The Journal of Arthroplasty, vol. 23, no. 7, pp. 984-991, 2008.

[6] H.-R. Choi, F. Von Knoch, D. Zurakowski, S. B. Nelson, and H. Malchau, "Can implant retention be recommended for treatment of infected TKA?" Clinical Orthopaedics and Related Research, vol. 469, no. 4, pp. 961-969, 2011.

[7] D. T. Tsukayama, R. Estrada, and R. B. Gustilo, "Infection after total hip arthroplasty: a study of the treatment of one hundred and six infections," The Journal of Bone \& Joint Surgery, vol. 78, no. 4, pp. 512-523, 1996.
[8] M. Faschingbauer, T. Kappe, A. Trubrich, R. Bieger, and H. Reichel, "Retention of the prosthesis in early periprosthetic infection after total hip arthroplasty," Zeitschrift für Orthopädie und Unfallchirurgie, vol. 153, no. 2, pp. 192-197, 2015.

[9] K. A. Azzam, M. Seeley, E. Ghanem, M. S. Austin, J. J. Purtill, and J. Parvizi, "Irrigation and debridement in the management of prosthetic joint infection: traditional indications revisited," The Journal of Arthroplasty, vol. 25, no. 7, pp. 1022-1027, 2010.

[10] T. K. Fehring, S. M. Odum, K. R. Berend et al., "Failure of Irrigation and Débridement for Early Postoperative Periprosthetic Infection," Clinical Orthopaedics and Related Research, vol. 471, no. 1, pp. 250-257, 2013.

[11] F. S. Haddad, M. Sukeik, and S. Alazzawi, "Is Single-stage Revision According to a Strict Protocol Effective in Treatment of Chronic Knee Arthroplasty Infections?" Clinical Orthopaedics and Related Research, vol. 473, no. 1, pp. 8-14, 2015.

[12] D. Kendoff and T. Gehrke, "Surgical management of periprosthetic joint infection: one-stage exchange," The Journal of Knee Surgery, vol. 27, no. 4, pp. 273-278, 2014.

[13] T. Gehrke and D. Kendoff, "Peri-Prosthetic Hip Infections: In Favour of One-Stage," Hip International, vol. 22, no. 8_suppl, pp. 40-45, 2012.

[14] T. K. Fehring, S. Odum, T. F. Calton, and J. B. Mason, "Articulating versus static spacers in revision total knee arthroplasty for sepsis," Clinical Orthopaedics and Related Research, no. 380, pp. 9-16, 2000.

[15] G. S. Van Thiel, K. R. Berend, G. R. Klein, A. C. Gordon, A. V. Lombardi, and C. J. Della Valle, "Intraoperative molds to create an articulating spacer for the infected knee arthroplasty," Clinical Orthopaedics and Related Research, vol. 469, no. 4, pp. 994-1001, 2011.

[16] M. G. Freeman, T. K. Fehring, S. M. Odum, K. Fehring, W. L. Griffin, and J. B. Mason, "Functional Advantage of Articulating Versus Static Spacers in 2-Stage Revision for Total Knee Arthroplasty Infection," The Journal of Arthroplasty, vol. 22, no. 8, pp. 1116-1121, 2007.

[17] A. A. Haleem, D. J. Berry, and A. D. Hanssen, "Mid-term to long-term followup of two-stage reimplantation for infected total knee arthroplasty," Clinical Orthopaedics and Related Research, no. 428, pp. 35-39, 2004.

[18] A. A. Hofmann, T. D. Goldberg, A. M. Tanner, and T. M. Cook, "Ten-year experience using an articulating antibiotic cement hip spacer for the treatment of chronically infected total hip," The Journal of Arthroplasty, vol. 20, no. 7, pp. 874-879, 2005.

[19] W. J. Hart and R. S. Jones, "Two-stage revision of infected total knee replacements using articulating cement spacers and shortterm antibiotic therapy," The Journal of Bone \& Joint Surgery (British Volume), vol. 88, no. 8, pp. 1011-1015, 2006.

[20] D. R. Osmon, E. F. Berbari, A. R. Berendt et al., "Executive summary: Diagnosis and management of prosthetic joint infection: 
Clinical practice guidelines by the infectious diseases society of america," Clinical Infectious Diseases, vol. 56, no. 1, pp. 1-10, 2013.

[21] W. Zimmerli, A. Trampuz, and P. E. Ochsner, "Current concepts: prosthetic-joint infections," The New England Journal of Medicine, vol. 351, no. 16, pp. 1645-1654, 2004.

[22] K. H. Wang, S. W. Yu, R. Iorio, A. J. Marcantonio, and M. S. Kain, "Long Term Treatment Results for Deep Infections of Total Knee Arthroplasty," The Journal of Arthroplasty, vol. 30, no. 9, pp. 1623-1628, 2015.

[23] L. A. Whiteside, T. A. Nayfeh, R. LaZear, and M. E. Roy, "Reinfected Revised TKA Resolves With an Aggressive Protocol and Antibiotic Infusion," Clinical Orthopaedics and Related Research, vol. 470, no. 1, pp. 236-243, 2012.

[24] J. Stammers, S. Kahane, V. Ranawat et al., "Outcomes of infected revision knee arthroplasty managed by two-stage revision in a tertiary referral centre," The Knee, vol. 22, no. 1, pp. 56-62, 2015.

[25] J. C. Sherrell, T. K. Fehring, and S. Odum, "The chitranjan ranawat award: fate of two-stage reimplantation after failed irrigation and débridement for periprosthetic knee infection," Clinical Orthopaedics and Related Research, vol. 469, no. 1, pp. 18-25, 2011.

[26] M. W. Pagnano, R. T. Trousdale, and A. D. Hanssen, "Outcome after reinfection following reimplantation hip arthroplasty," Clinical Orthopaedics and Related Research, no. 338, pp. 192204, 1997.

[27] T. Winkler, A. Trampuz, S. Hardt, V. Janz, C. Kleber, and C. Perka, "Periprosthetic infection after hip arthroplasty," Der Orthopäde, vol. 43, no. 1, pp. 70-78, 2014.

[28] J. Parvizi and T. Gehrke, "Definition of periprosthetic joint infection," The Journal of Arthroplasty, vol. 29, no. 7, p. 1331, 2014.

[29] M. Faschingbauer, H. Reichel, R. Bieger, and T. Kappe, "Mechanical complications with one hundred and thirty eight (antibiotic-laden) cement spacers in the treatment of periprosthetic infection after total hip arthroplasty," International Orthopaedics, vol. 39, no. 5, pp. 989-994, 2015.

[30] M. Faschingbauer, R. Bieger, H. Reichel, C. Weiner, and T. Kappe, "Complications associated with 133 static, antibioticladen spacers after TKA," Knee Surgery, Sports Traumatology, Arthroscopy, vol. 24, no. 10, pp. 3096-3099, 2016.

[31] J. Kelm, E. Schmitt, and K. Anagnostakos, "Vacuum-assisted closure in the treatment of early hip joint infections," International Journal of Medical Sciences, vol. 6, no. 5, pp. 241-246, 2009.

[32] W. A. Jiranek, A. C. Waligora, S. R. Hess, and G. L. Golladay, "Surgical treatment of prosthetic joint infections of the hip and knee: changing paradigms?" The Journal of Arthroplasty, vol. 30, no. 6, pp. 912-918, 2015.

[33] H. A. C. Leonard, A. D. Liddle, Ó. Burke, D. W. Murray, and H. Pandit, "Single- or two-stage revision for infected total hip arthroplasty? A systematic review of the literature," Clinical Orthopaedics and Related Research, vol. 472, no. 3, pp. 10361042, 2014.

[34] J. P. M. Masters, N. A. Smith, P. Foguet, M. Reed, H. Parsons, and A. P. Sprowson, "A systematic review of the evidence for single stage and two stage revision of infected knee replacement," $B M C$ Musculoskeletal Disorders, vol. 14, article no. 222, 2013.

[35] B. Waagsbø, A. Sundøy, T. M. L. Martinsen, and L. S. Nymo, "Treatment results with debridement and retention of infected hip prostheses," Infectious Diseases, vol. 41, no. 8, pp. 563-568, 2009.

[36] J. R. Crockarell Jr., A. D. Hanssen, D. R. Osmon, and B. F. Morrey, "Treatment of infection with debridement and retention of the components following hip arthroplasty," The Journal of Bone \& Joint Surgery, vol. 80, no. 9, pp. 1306-1313, 1998.

[37] C. H. Wu, C. F. Gray, and G.-C. Lee, "Arthrodesis Should Be Strongly Considered After Failed Two-stage Reimplantation TKA," Clinical Orthopaedics and Related Research, vol. 472, no. 11, pp. 3295-3304, 2014.

[38] T. N. Peel, K. L. Buising, M. M. Dowsey et al., "Outcome of debridement and retention in prosthetic joint infections by methicillin-resistant staphylococci, with special reference to rifampin and fusidic acid combination therapy," Antimicrobial Agents and Chemotherapy, vol. 57, no. 1, pp. 350-355, 2013.

[39] J. Lora-Tamayo, O. Murillo, J. A. Iribarren et al., "A large multicenter study of methicillin-susceptible and methicillin-resistant staphylococcus aureus prosthetic joint infections managed with implant retention," Clinical Infectious Diseases, vol. 56, no. 2, pp. 182-194, 2013.

[40] F. Vilchez, J. C. Martínez-Pastor, S. García-Ramiro et al., "Outcome and predictors of treatment failure in early post-surgical prosthetic joint infections due to Staphylococcus aureus treated with debridement," Clinical Microbiology and Infection, vol. 17, no. 3, pp. 439-444, 2011.

[41] A.-P. Puhto, T. M. Puhto, T. T. Niinimäki, J. I. Leppilahti, and H. P. T. Syrjälä, "Two-stage revision for prosthetic joint infection: outcome and role of reimplantation microbiology in 107 cases," The Journal of Arthroplasty, vol. 29, no. 6, pp. 1101-1104, 2014.

[42] F. S. Haddad, S. K. Muirhead-Allwood, A. R. J. Manktelow, and I. Bacarese-Hamilton, "Two-stage uncemented revision hip arthroplasty for infection," The Journal of Bone \& Joint Surgery (British Volume), vol. 82, no. 5, pp. 689-694, 2000.

[43] B. Zmistowski, M. W. Tetreault, P. Alijanipour, A. F. Chen, C. J. Della Valle, and J. Parvizi, "Recurrent periprosthetic joint infection: Persistent or new infection?" The Journal of Arthroplasty, vol. 28, no. 9, pp. 1486-1489, 2013.

[44] M. J. Kraay, V. M. Goldberg, S. J. Fitzgerald, and M. J. Salata, "Cementless two-staged total hip arthroplasty for deep periprosthetic infection," Clinical Orthopaedics and Related Research, no. 441, pp. 243-249, 2005.

[45] G. K. Triantafyllopoulos, S. G. Memtsoudis, W. Zhang, Y. Ma, T. P. Sculco, and L. A. Poultsides, "Periprosthetic Infection Recurrence After 2-Stage Exchange Arthroplasty: Failure or Fate?" The Journal of Arthroplasty, vol. 32, no. 2, pp. 526-531, 2017. 


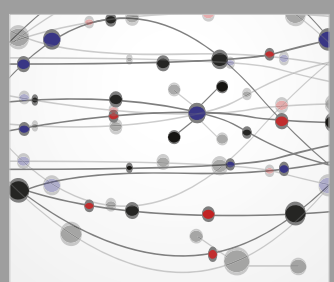

The Scientific World Journal
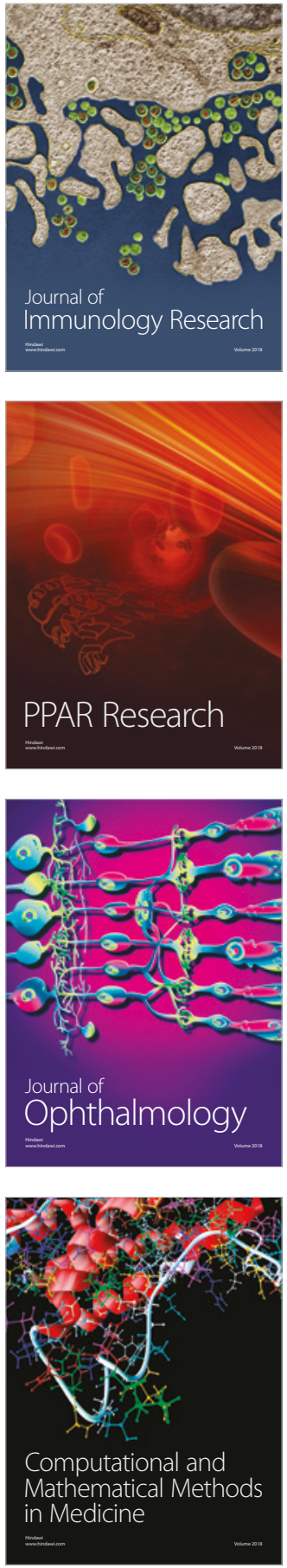

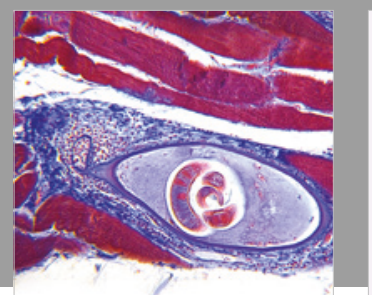

Gastroenterology Research and Practice

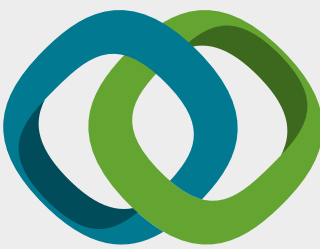

\section{Hindawi}

Submit your manuscripts at

www.hindawi.com
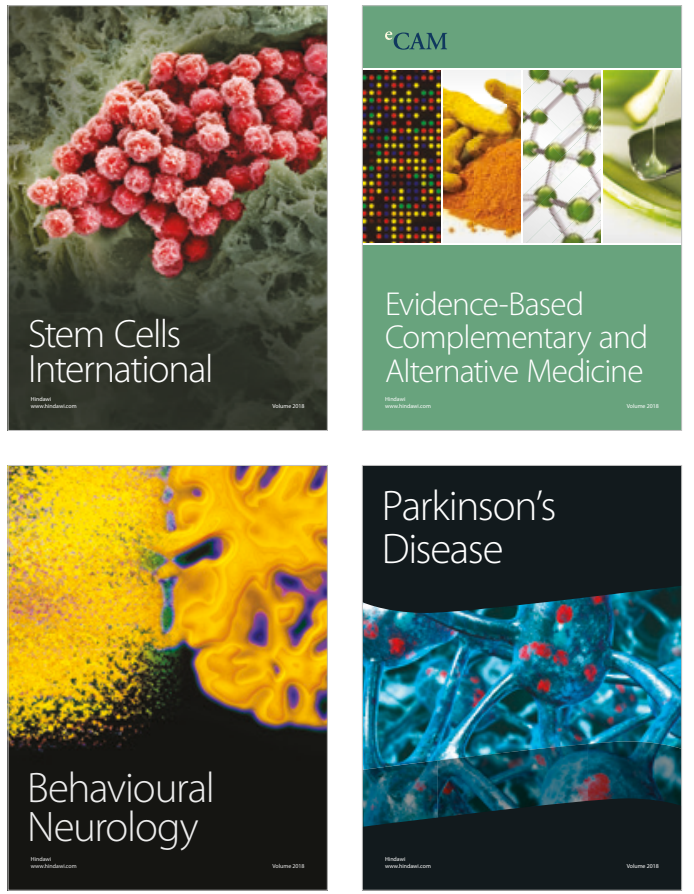

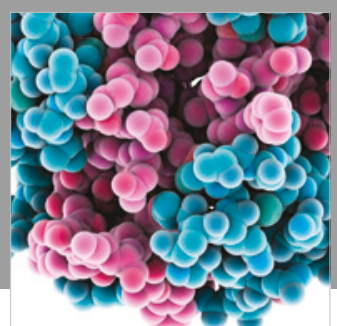

ournal of

Diabetes Research

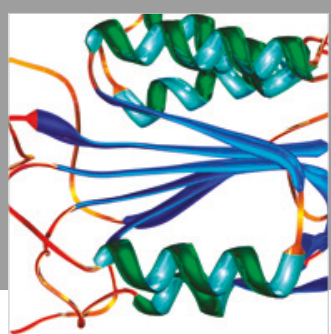

Disease Markers
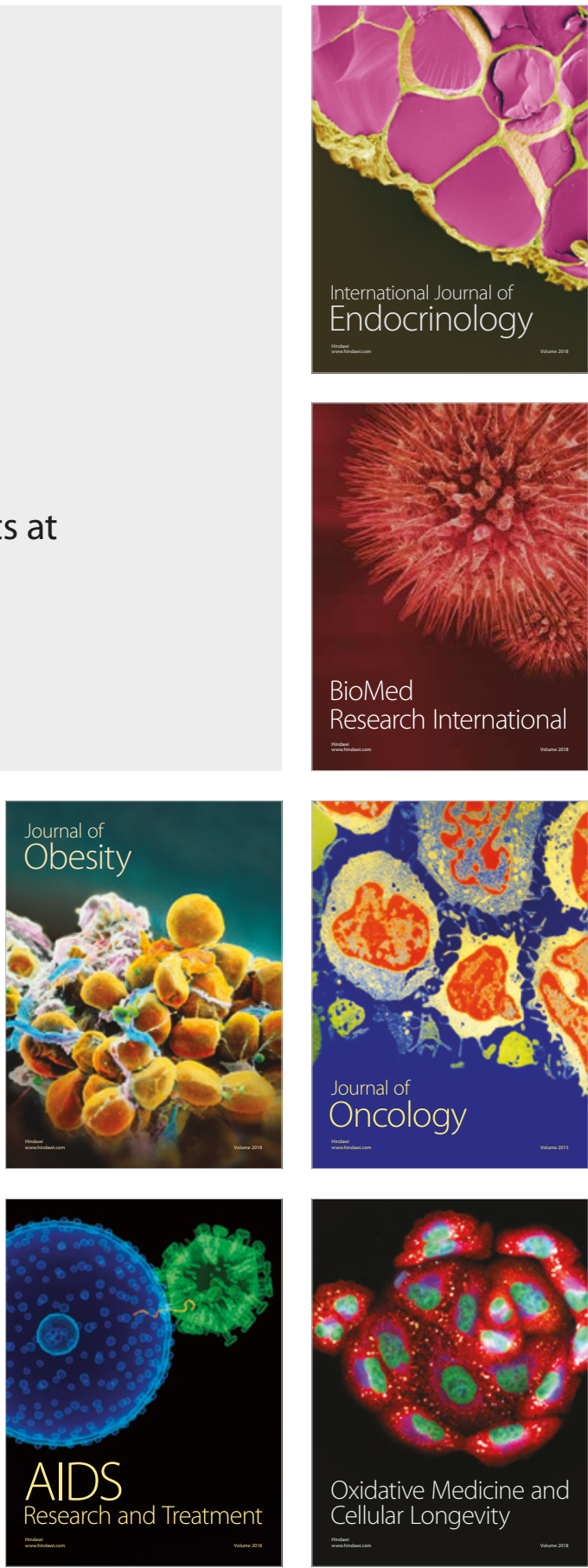